\title{
Development of Modified Fractional Fluid Flow Equation For Non - Darcy Flow in Computer Simulation of Oil Reservoirs
}

\author{
${ }^{* 1}$ Akintola, S. A., ${ }^{2}$ Adeaga, O. A. \& ${ }^{3}$ Muritala, O. K. \\ *1,3Department of Petroleum Engineering, University of Ibadan, Ibadan. Oyo State. Nigeria. \\ 2Department of Mechanical Engineering, First Technical University, Ibadan. Oyo State. Nigeria \\ Corresponding Author: sarah.akintola@ui.edu.ng (+2348023363651)
}

\begin{abstract}
Upon the depletion of oil reservoir, huge amount of oil is usually left behind. This oil, in some cases double the initial oil recovered, in order to recover the unrecovered oil, different types of secondary oil recovery techniques can be explored. A more common techniques is water flooding which involve the injection of water into reservoir to displace oil into the wellbore. To determine the relative flow rates of oil and water at any point in a porous flow system while also examining factors such as fluid properties, rock properties, reservoir structural properties, pressure gradient, and flow rate which affect the displacement efficiency of a water flooding project, the fractional flow equation is employed. But the convectional fractional flow equation is applicable just to Darcy flow. The use of Darcy flow equation is not applicable in low permeability sandstone reservoir, hence non Darcy flow have been used one of such equation is the Forcheimer equation, as a result this study is aimed at modifying the Forcheimer equation and validating the new fractional flow equation using literature. The result obtained showed that the proposed equation predicts better than Forcheimer equation
\end{abstract}

Keyword: Darcy flow, Forcheimer equation, water flooding fractional flow equation,

Proceedings Reference Format

Akintola, S. A., Adeaga, O. A. \& Muritala, O. K. (2021): Development of Modified Fractional Fluid Flow Equation For Non - Darcy Flow in Computer Simulation of Oil Reservoirs. Proceedings of the 27th iSTEAMS Multidisciplinary Innovations \& Technology Transfer (MINTT) Conference. Academic City University College, Accra, Ghana. June, 2021. Pp 407-416 www.isteams.net/ghana2021.

DOI - https://doi.org/ 10.22624/AIMS/iSTEAMS-2021/N27P38

\section{INTRODUCTION}

It is generally acknowledged that the first water flooding occurred as a result of an accidental water injection in the Pothoic city area of Pennsylvania in 1865. In 1880 John F Carll concluded that oil recovery might be increased by the injection of water into the reservoir to displace oil to producing wells. Primary oil recovery under natural producing mechanisms (i.e. liquid and rock expansion, solution gas drives, gas cap drive and natural water influx) leaves behind $50 \%$ to $80 \%$ of the original oil in place consequently, a vast amount of oil remains unrecovered. A rule-of-thumb is that water flooding will recover half-again as much oil as was produced under primary. Water flooding is known to be one of the economical and widely used post-primary recovery method for light-to medium-oil reservoirs. Water flooding is usually a secondary drive mechanism initiated before or after the depletion of the primary drive mechanism of the reservoirs. 
Prediction and understanding of water flooding performance began with the Fractional Flow Curve (FFC), where fractional flow (for Darcy) equation and capillary pressure gradient is used to show the relationship between fraction of water flowing in the reservoir and water saturation. The fractional flow equation uses Darcy's Law to relate the fraction of displacing fluid to the total flow stream, at any point in the reservoir. Darcy's Law (equation 1.0) was applied at low flow rate (Laminar flow) which are found throughout the oil reservoir.

$q=-\frac{K A}{\mu} \frac{\delta P}{\delta S}$

And Darcy's law state that the velocity of a homogeneous fluid in a porous medium is proportional to the pressure gradient and inversely proportional to the fluid viscosity, which is presented in equation 2.0

$v=-\frac{k}{\mu} \frac{\delta p}{\delta s}$

Buckley Leverett (1941) used the concept of Fractional flow, beginning with Darcy's law for water and oil in a 1-D flow, formulated the Fractional flow equation presented in equation 3.0

$f_{w}=\frac{1+\frac{0.001127 k_{0} A}{\mu_{0} q_{t}}\left[\frac{\partial P_{c}}{\partial x}-0.00694\left(\rho_{w}-\rho_{0}\right) \sin \sin \theta\right]}{1+\frac{\mu_{W} K_{0}}{\mu_{0} k_{w}}}$

Forchheimer, $\mathrm{P}$ (1901) was the first to suggest a non-linear relationship between hydraulic gradient and flux at large Reynold number and Zeng et al (2001) on non-Darcy flow in low permeable sandstones reservoir. The inertia effect takes the form of distorted flow path and turbulent flow of different location in the reservoir. This effect is accounted for in the Forcheimer fractional flow equation (equation 4.0).

$-\frac{\delta P}{\delta S}=\frac{\mu V}{k}+F_{t} P V^{2}$

Li and et al., (2001) perform experiment on Berea sandstone core to stimulate wafer non- Darcy flow proposed a correlation for predicting Forcheirmer coefficient as given in equation 5.0

$k^{\prime}=\frac{k \emptyset}{11500}$

\section{METHODOLOGY}

According to Darcy's flow equation; qw is presented in the equation 6.0

$\mathrm{q}_{\mathrm{w}}=-0.001127 \frac{K_{w} A}{\mu_{w}}\left[\frac{\delta P_{w}}{\delta s}+0.00694 \rho_{w} \sin \sin \theta\right]$

Modifying this for non-darcy flow, using Forcheimer equation to obtain the equation 7.0

$\frac{\delta p}{d s}=-\frac{\mu}{k} q-\frac{\rho}{k^{1}} q^{2}$ 
$\mathrm{q}_{\mathrm{w}}=-0.001127 \frac{K_{w} A}{\mu_{w}}\left[\frac{\mu_{w}}{k_{w}} q_{w}-\frac{\rho_{w}}{k^{1} w} q^{2}{ }_{w}+0.00694 \rho_{w} \sin \sin \theta\right]$.

Rearranging and collecting the water phase and the oil phase, the equation 9.0 and 10.0 , respectively, is developed.

$\left[-\frac{\mu_{w}}{k_{w}} q_{w}-\frac{\rho_{w}}{k^{1} w} q_{w}^{2}\right]=-\frac{q_{w} \mu_{w}}{0.001127 k_{w} A}-0.00694 \rho_{w} \sin \sin \theta$

For oil phase;

$-\left[\frac{\mu_{o}}{k_{o}} q_{o}-\frac{\rho_{o}}{k^{1} o} q_{o}^{2}\right]=-\frac{q_{o} \mu_{o}}{0.001127 k_{o} A}-0.00694 \rho_{o}(8.0)$

But capillary pressure, $P_{C}=P_{O}-P_{W}$

Applying the equation 10.0a Thus;

$\left[-\frac{\mu_{o}}{k_{o}} q_{o}-\frac{\rho_{o}}{k^{1} o} q_{o}^{2}\right]-\frac{\mu_{w}}{k_{w}} q_{w}-\frac{\rho_{w}}{k^{1} w} q_{w}^{2}=\frac{q_{w} \mu_{w}}{0.001127 k_{w} A}-\frac{q_{o} \mu_{o}}{0.001127 k_{o} A}$ $+0.00694\left(\rho_{w}-\rho_{o}\right) \sin \sin \theta \ldots$

Given $q_{t}=q_{o}+q_{w}$

and. $q_{o}=q_{t}-q_{w}$

Applying the equations $11.0 \mathrm{a}$ and $11.0 \mathrm{~b}$ Thus;

$\left[-\frac{\mu_{o}}{k_{o}}\left(q_{t}-q_{w}\right)-\frac{\rho_{o}}{k^{1}{ }_{o}}\left(q_{t}-q_{w}\right)^{2}\right]-\left[-\frac{\mu_{w}}{k_{w}} q_{w}-\frac{\rho_{w}}{k^{1} w} q_{w}^{2}\right]$

$=\frac{q_{w} \mu_{w}}{0.001127 k_{w} A}-\frac{q_{o} \mu_{o}}{0.001127 k_{o} A}+0.00694\left(\rho_{w}-\rho_{o}\right) \sin \sin \theta$

$-\left[\frac{\mu_{o}}{k_{o}} q_{t}+\frac{\mu_{o}}{k_{o}} q_{w}\right]-\left[\frac{\rho_{o}}{k_{o}^{1}} q_{t}^{2}-2 \frac{\rho_{o}}{k_{o}^{1}} q_{t} q_{w}+\frac{\rho_{o}}{k_{o}^{1}} q_{w}^{2}\right]-\frac{\mu_{w}}{k_{w}} q_{w}-\frac{\rho_{w}}{k^{1} w} q_{w}^{2}$

$=\frac{q_{w} \mu_{w}}{0.001127 k_{w} A}-\frac{q_{o} \mu_{o}}{0.001127 k_{o} A}+0.00694\left(\rho_{w}-\rho_{o}\right) \sin \sin \theta \ldots$

$-\frac{\mu_{o}}{k_{o}} q_{t}+\frac{\mu_{o}}{k_{o}} q_{w}-\frac{\rho_{o}}{k_{o}^{1}} q_{t}^{2}+2 \frac{\rho_{o}}{k_{o}^{1}} q_{t} q_{w}-\frac{\rho_{o}}{k_{o}^{1}} q_{w}^{2}+\frac{\mu_{w}}{k_{w}} q_{w}+\frac{\rho_{w}}{k^{1} w} q_{w}^{2}$

$=\frac{q_{w} \mu_{w}}{0.00112{ }_{w} A}-\frac{\left(q_{t}-q_{w}\right) \mu_{o}}{0.001127 k_{o} A}+0.00694\left(\rho_{w}-\rho_{o}\right) \sin \sin \theta$ 
Collecting like terms;

$2 \frac{\rho_{o}}{k_{o}^{1}} q_{t} q_{w}+\frac{\mu_{o}}{k_{o}} q_{w}-\frac{\rho_{o}}{k_{o}^{1}} q_{w}^{2}+\frac{\mu_{w}}{k_{w}} q_{w}+\frac{\rho_{w}}{k^{1} w} q_{w}^{2}-\frac{q_{w} \mu_{w}}{0.001127 k_{w} A}-\frac{q_{w} \mu_{o}}{0.001127 k_{w} A}$

$=\frac{\rho_{o}}{k_{o}^{1}} q_{t}^{2}-\frac{\mu_{o}}{k_{o}} q_{t}+\frac{q_{t} \mu_{o}}{0.001127 k_{w} A}+0.00694\left(\rho_{w}-\rho_{o}\right) \sin \sin \theta$

$\left(\frac{\rho_{w}}{k_{w}^{1}}-\frac{\rho_{o}}{k_{o}^{1}}\right) q_{w}^{2}+\left(2 \frac{\rho_{o}}{k_{o}^{1}} q_{t}+\frac{\mu_{o}}{k_{o}}+\frac{\mu_{w}}{k_{w}}-\frac{\mu_{w}}{0.001127 k_{w} A}-\frac{\mu_{o}}{0.001127 k_{w} A}\right) q_{w}$

$+\left(-\frac{\mu_{o}}{k_{o}} q_{t}+\frac{q_{t} \mu_{o}}{0.001127 k_{w} A}-\frac{\rho_{o}}{k_{o}^{1}} q_{t}^{2}-0.00694\left(\rho_{w}-\rho_{o}\right) \sin \sin \theta\right)=0 \ldots$

Applying quadratic formula 17.0;

$q_{w}=\frac{-\beta \pm \sqrt{\beta^{2}-4(\alpha)(\gamma)}}{2 \alpha}$

Where $\alpha=\left(\frac{\rho_{w}}{k_{w}^{1}}-\frac{\rho_{o}}{k_{o}^{1}}\right) ; \beta=\left(2 \frac{\rho_{o}}{k_{o}^{1}} q_{t}+\frac{\mu_{o}}{k_{o}}+\frac{\mu_{w}}{k_{w}}-\frac{\mu_{w}}{0.001127 k_{w} A}-\frac{\mu_{o}}{0.001127 k_{w} A}\right)$

$$
\gamma=\left(-\frac{\mu_{o}}{k_{o}} q_{t}+\frac{q_{t} \mu_{o}}{0.001127 k_{w} A}-\frac{\rho_{o}}{k_{o}^{1}} q_{t}^{2}-0.00694\left(\rho_{w}-\rho_{o}\right) \sin \sin \theta\right)
$$

Thus, $f_{w}=\frac{q_{w}}{q_{t}}$

equation 19.0 present the new fractional flow equation

$f_{w}=\frac{-\beta}{2 q_{t} \alpha} \pm \frac{1}{2 \alpha q_{t}} \sqrt{\beta^{2}-4 \alpha \gamma}$ 


\section{RESULTS AND DISCUSSION}

The data obtained from James T. Smith, Water flooding (1997) were used for the input data for the two case scenarios. For Case 1.0, the Tables 1.0, 2.0, 3.0 and 4.0, presents the data for Relative Permeability and Water Saturation; Buckley Leverett Fractional Flow; Modified Fractional Flow and Modified Fractional Flow respectively. While that for case 2.0 input data for Relative Permeability and Water Saturation Data, Buckley Leverett Fractional Flow, :Modified Fractional Flow and Modified Fractional Flow are presented in the Tables 5.0, 6.0, 7.0 and 8.0, respectively. The flow chart used in the validation of the Modified Fractional Flow Equation is presented in the fig. 1.0

As seen in the Figs. 2.0 and 3.0, the Modified Fractional Flow Curve attained earlier water breakthrough than that of the Buckley Leverett. This can be attributed to the turbulence effect created by the Non Darcy Flow which will influence the Fractional Flow Curve. The initial slow progressive rate of displacing fluid is due to low permeability and as the turbulence takes effect it then shoot up sharply.

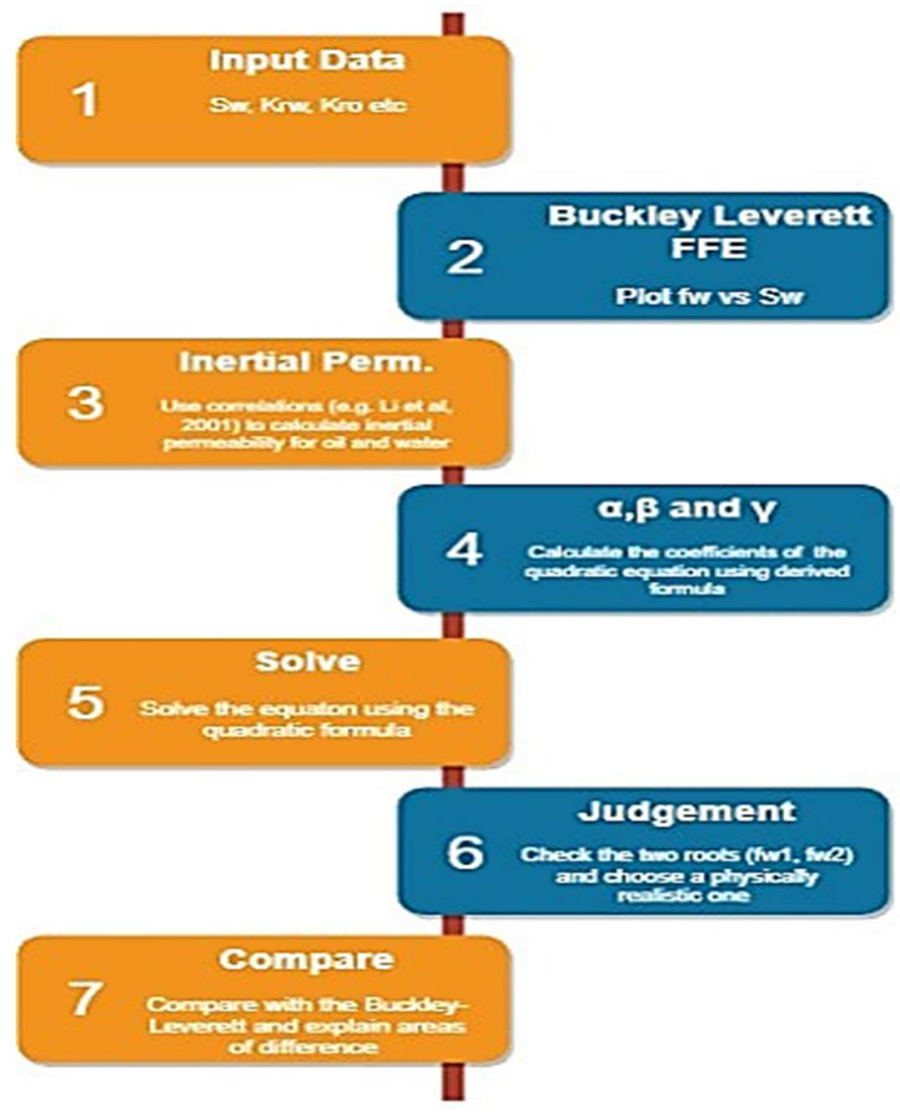

Fig. 1.0: Flow chart for the validation of the Modified Fractional Flow Equation 
CASE 1: INPUT DATA (Source: James T. Smith, Water flooding 1997)

Table 1.0: Relative Permeability and Water Saturation Data

\begin{tabular}{|l|l|l|l|}
\hline Reservoir Data & \multicolumn{3}{|l|}{ Relative Permeability and Water Saturation Data } \\
\hline Average Porosity $=25 \%$ & $\mathrm{~S}_{\mathrm{w}}$ & $\mathrm{K}_{\mathrm{r}}$ & $\mathrm{K}_{\mathrm{rw}}$ \\
\hline Absolute Permeability $=50 \mathrm{mD}$ & 0.2 & 0.85 & 0 \\
\hline Density of Water $=62.4 \mathrm{lbm} / \mathrm{cc}$ & 0.25 & 0.8 & 0.002 \\
\hline Density of oil $=55 \mathrm{lbm} / \mathrm{cc}$ & 0.3 & 0.61 & 0.009 \\
\hline Viscosity of oil $=\mu_{0}=6.5 \mathrm{cp}$ & 0.35 & 0.47 & 0.018 \\
\hline Viscosity of water $=\mu_{w}=0.9 \mathrm{cp}$ & 0.4 & 0.37 & 0.029 \\
\hline Average Porosity $=25 \%$ & 0.45 & 0.285 & 0.044 \\
\hline $\mathrm{i}_{\mathrm{w}}=1000 \mathrm{RB} / \mathrm{D}$ & 0.5 & 0.22 & 0.064 \\
\hline $\mathrm{E}_{\mathrm{A}}=100 \%$ & 0.55 & 0.163 & 0.086 \\
& & & \\
\hline $\mathrm{q}_{\mathrm{t}}=1000 \mathrm{RB} / \mathrm{D}$ & 0.6 & 0.12 & 0.117 \\
\hline Area $=10$ acres & 0.65 & 0.081 & 0.152 \\
\hline & 0.7 & 0.05 & 0.19 \\
\cline { 2 - 4 } & 0.75 & 0.027 & 0.232 \\
\cline { 2 - 4 } & 0.8 & 0.01 & 0.247 \\
\cline { 2 - 4 } & 0.85 & 0 & 0.25 \\
\hline
\end{tabular}

Table 2.0: Buckley Leverett Fractional Flow

\begin{tabular}{|r|r|r|r|r|}
\hline \multicolumn{1}{|l|}{$\mathbf{S}_{\mathrm{w}}$} & $\mathbf{K}_{\mathrm{ro}}$ & $\mathbf{K}_{\mathrm{rw}}$ & $\mathbf{K}_{\mathrm{ro}} / \mathbf{k}_{\mathrm{rw}}$ & $\mathbf{F}_{\mathrm{w}}$ \\
\hline 0.2 & 0.85 & 0 & 400 & 0.017735 \\
\hline 0.25 & 0.8 & 0.002 & 67.77778 & 0.096296 \\
\hline 0.3 & 0.61 & 0.009 & 26.11111 & 0.216667 \\
\hline 0.35 & 0.47 & 0.018 & 12.75862 & 0.361457 \\
\hline 0.4 & 0.37 & 0.029 & 6.477273 & 0.527189 \\
\hline 0.45 & 0.285 & 0.044 & 3.4375 & 0.677524 \\
\hline 0.5 & 0.22 & 0.064 & 1.895349 & 0.792121 \\
\hline 0.55 & 0.163 & 0.086 & 1.025641 & 0.875648 \\
\hline 0.6 & 0.12 & 0.117 & 0.532895 & 0.931285 \\
\hline 0.65 & 0.081 & 0.152 & 0.263158 & 0.964844 \\
\hline 0.7 & 0.05 & 0.19 & 0.116379 & 0.984141 \\
\hline 0.75 & 0.027 & 0.232 & 0.040486 & 0.994426 \\
\hline 0.8 & 0.01 & 0.247 & & \\
\hline
\end{tabular}


Table 3.0: Modified Fractional Flow

\begin{tabular}{|l|l|l|l|l|}
\hline \multicolumn{1}{|c|}{$\mathbf{S}_{\mathbf{w}}$} & $\mathbf{K}_{\mathrm{ro}}$ & $\mathrm{K}_{\mathrm{rw}}$ & $\mathbf{K}_{\mathbf{w}}{ }^{1}$ & $\mathrm{~K}_{\mathbf{1}}{ }^{1}$ \\
\hline 0.2 & 0.85 & 0 & - & $\mathbf{0 . 0 0 0 9}$ \\
\hline 0.25 & 0.8 & 0.002 & $\mathbf{0 . 0 0 0 0 0}$ & $\mathbf{0 . 0 0 0 9}$ \\
\hline 0.3 & 0.61 & 0.009 & $\mathbf{0 . 0 0 0 0 1}$ & $\mathbf{0 . 0 0 0 7}$ \\
\hline 0.35 & 0.47 & 0.018 & $\mathbf{0 . 0 0 0 0 2}$ & $\mathbf{0 . 0 0 0 5}$ \\
\hline 0.4 & 0.37 & 0.029 & $\mathbf{0 . 0 0 0 0 3}$ & $\mathbf{0 . 0 0 0 4}$ \\
\hline 0.45 & 0.285 & 0.044 & $\mathbf{0 . 0 0 0 0 5}$ & $\mathbf{0 . 0 0 0 3}$ \\
\hline 0.5 & 0.22 & 0.064 & $\mathbf{0 . 0 0 0 0 7}$ & $\mathbf{0 . 0 0 0 2}$ \\
\hline 0.55 & 0.163 & 0.086 & $\mathbf{0 . 0 0 0 0 9}$ & $\mathbf{0 . 0 0 0 2}$ \\
\hline 0.6 & 0.12 & 0.117 & $\mathbf{0 . 0 0 0 1 3}$ & $\mathbf{0 . 0 0 0 1}$ \\
\hline 0.65 & 0.081 & 0.152 & $\mathbf{0 . 0 0 0 1 7}$ & $\mathbf{0 . 0 0 0 1}$ \\
\hline 0.7 & 0.05 & 0.19 & $\mathbf{0 . 0 0 0 2 1}$ & $\mathbf{0 . 0 0 0 1}$ \\
\hline 0.75 & 0.027 & 0.232 & $\mathbf{0 . 0 0 0 2 5}$ & $\mathbf{0 . 0 0 0 0}$ \\
\hline 0.8 & 0.01 & 0.247 & $\mathbf{0 . 0 0 0 2 7}$ & $\mathbf{0 . 0 0 0 0}$ \\
\hline
\end{tabular}

Table 4.0: Modified Fractional Flow

\begin{tabular}{|r|r|r|r|}
\hline \multicolumn{1}{|c|}{$\boldsymbol{\beta}$} & $\boldsymbol{\gamma}$ & $\boldsymbol{\gamma}$ & $\mathbf{F}_{\mathrm{w}}{ }^{1}$ \\
\hline 0.0 & 0.0 & 0.0 & 0.0 \\
\hline 28640750 & 815693.1 & $7,469,232,476.66)$ & 0.016135 \\
\hline 6295716 & 147854.6 & -9801997017 & 0.039446 \\
\hline 3081674 & 2152463 & -12722763422 & 0.063905 \\
\hline 1842829 & 13675224 & -16161764419 & 0.090012 \\
\hline 1127183 & 88474.34 & -20982193980 & 0.136397 \\
\hline 667000 & -204.319 & -27181637947 & 0.201872 \\
\hline 357105.4 & -151.693 & -36686982436 & 0.320522 \\
\hline 69000 & -111.004 & -49833234743 & 0.849837 \\
\hline-247007 & -84.6728 & -73827084605 & 1 \\
\hline
\end{tabular}




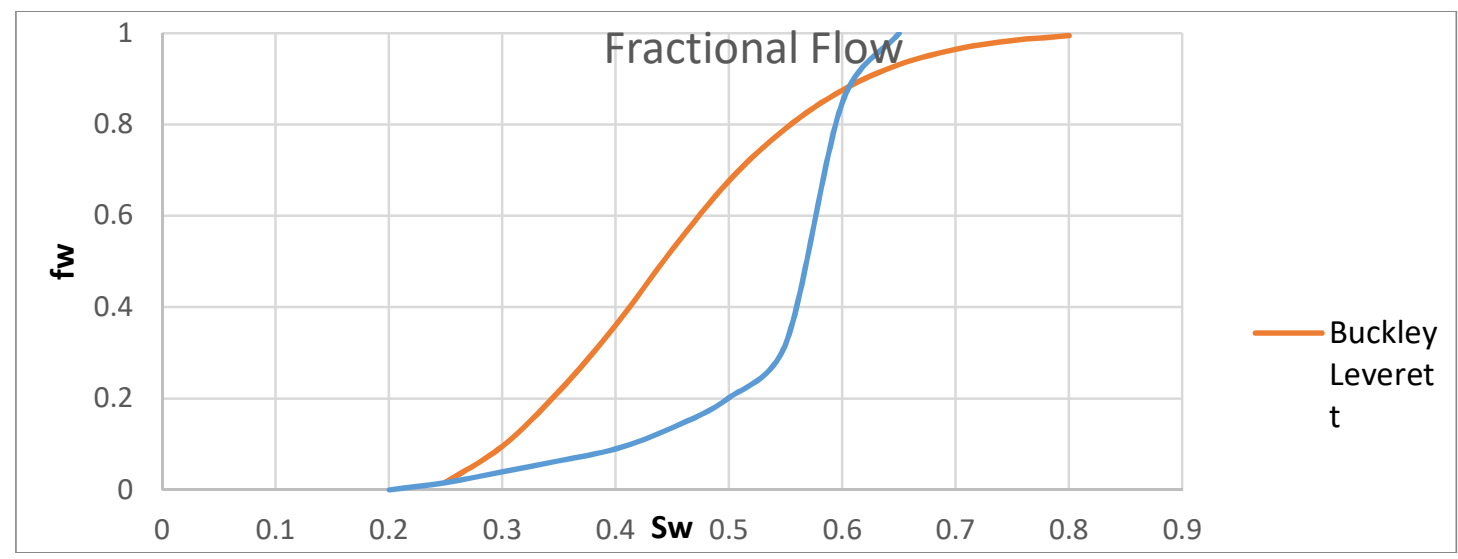

Figure. 1.0: Fractional flow of This model against the Buckley Leverett Method

CASE 2: INPUT DATA (Source: James T. Smith, Waterflooding 1997)

Table 5.0: Relative Permeability and Water Saturation Data

\begin{tabular}{|l|l|l|l|}
\hline Reservoir Data & \multicolumn{4}{l|}{ Relative Permeability and Water Saturation Data } \\
\hline Average Porosity $=18 \%$ & $\mathbf{S}_{\mathrm{w}}$ & $\mathbf{K}_{\mathrm{ro}}$ & $\mathrm{K}_{\mathrm{rw}}$ \\
\hline Absolute Permeability $=50 \mathrm{mD}$ & 0.2 & 0.93 & 0 \\
\hline Density of Water $=62.4 \mathrm{lbm} / \mathrm{cc}$ & 0.3 & 0.6 & 0.024 \\
\hline Density of oil $=55 \mathrm{lbm} / \mathrm{cc}$ & 0.4 & 0.36 & 0.045 \\
\hline Viscosity of oil $=\mu_{0}=2.48 c p$ & 0.5 & 0.228 & 0.124 \\
\hline Viscosity of water $=\mu_{w}=0.62 c p$ & 0.55 & 0.172 & 0.168 \\
\hline $\mathrm{i}_{\mathrm{w}}=1000 \mathrm{RB} / \mathrm{D}$ & 0.6 & 0.128 & 0.222 \\
\hline $\mathrm{E}_{\mathrm{A}}=100 \%$ & 0.7 & 0.049 & 0.35 \\
\hline $\mathrm{q}_{\mathrm{t}}=1000 \mathrm{RB} / \mathrm{D}$ & 0.8 & 0.018 & 0.512 \\
\hline Area $=10$ acres & 0.85 & 0 & 0.6 \\
\hline Average Porosity $=18 \%$ & \multicolumn{2}{|}{} \\
\hline
\end{tabular}

Table 6.0: Buckley Leverett Fractional Flow

\begin{tabular}{|c|c|c|c|c|}
\hline$S_{w}$ & $\mathrm{~K}_{\mathrm{ro}}$ & $\mathrm{K}_{\mathrm{rw}}$ & $\mathrm{K}_{\mathrm{ro}} / \mathrm{k}_{\mathrm{rw}}$ & $F_{w}$ \\
\hline 0.2 & 0.93 & 0 & & 0 \\
\hline 0.3 & 0.6 & 0.024 & 25 & 0.137931 \\
\hline 0.4 & 0.36 & 0.045 & 8 & 0.333333 \\
\hline 0.5 & 0.228 & 0.124 & 1.83871 & 0.685083 \\
\hline 0.55 & 0.172 & 0.168 & 1.02381 & 0.796209 \\
\hline 0.6 & 0.128 & 0.222 & 0.576577 & 0.874016 \\
\hline 0.7 & 0.049 & 0.35 & 0.14 & 0.966184 \\
\hline 0.8 & 0.018 & 0.512 & 0.035156 & 0.991288 \\
\hline 0.85 & 0 & 0.6 & 0 & 1 \\
\hline
\end{tabular}


Table 7.0:Modified Fractional Flow

\begin{tabular}{|c|c|c|c|c|}
\hline$S_{w}$ & $\mathrm{~K}_{\mathrm{ro}}$ & $\mathrm{K}_{\mathrm{rw}}$ & $\mathrm{K}_{\mathrm{w}}{ }^{1}$ & $\mathrm{~K}_{0}{ }^{1}$ \\
\hline 0.2 & 0.93 & 0 & - & 0.00073 \\
\hline 0.3 & 0.6 & 0.024 & 0.00002 & 0.00047 \\
\hline 0.4 & 0.36 & 0.045 & 0.00004 & 0.00028 \\
\hline 0.5 & 0.228 & 0.124 & 0.00010 & 0.00018 \\
\hline 0.55 & 0.172 & 0.168 & 0.00013 & 0.00013 \\
\hline 0.6 & 0.128 & 0.222 & 0.00017 & 0.00010 \\
\hline 0.7 & 0.049 & 0.35 & 0.00027 & 0.00004 \\
\hline 0.8 & 0.018 & 0.512 & 0.00040 & 0.00001 \\
\hline 0.85 & 0 & 0.6 & 0.00047 & - \\
\hline
\end{tabular}

Table 8.0: Modified Fractional Flow

\begin{tabular}{|r|r|r|r|}
\hline$\alpha$ & $\beta$ & $\gamma$ & $\mathbf{F}_{\mathbf{w}}{ }^{1}$ \\
\hline- & - & - & - \\
\hline 3205093 & 580734.3 & $(5,281,298,103.99)$ & 0.040502 \\
\hline 176636 & 241946.1 & $(8,802,371,334.45)$ & 0.074643 \\
\hline 334774.9 & 6164673 & $(13,898,599,987.30)$ & 0.194756 \\
\hline 66011.44 & 40859141 & $(18,423,746,427.42)$ & 0.30279 \\
\hline-189886 & 197631.9 & $(24,756,924,619.90)$ & 1 \\
\hline
\end{tabular}

\section{Fractional Flow}

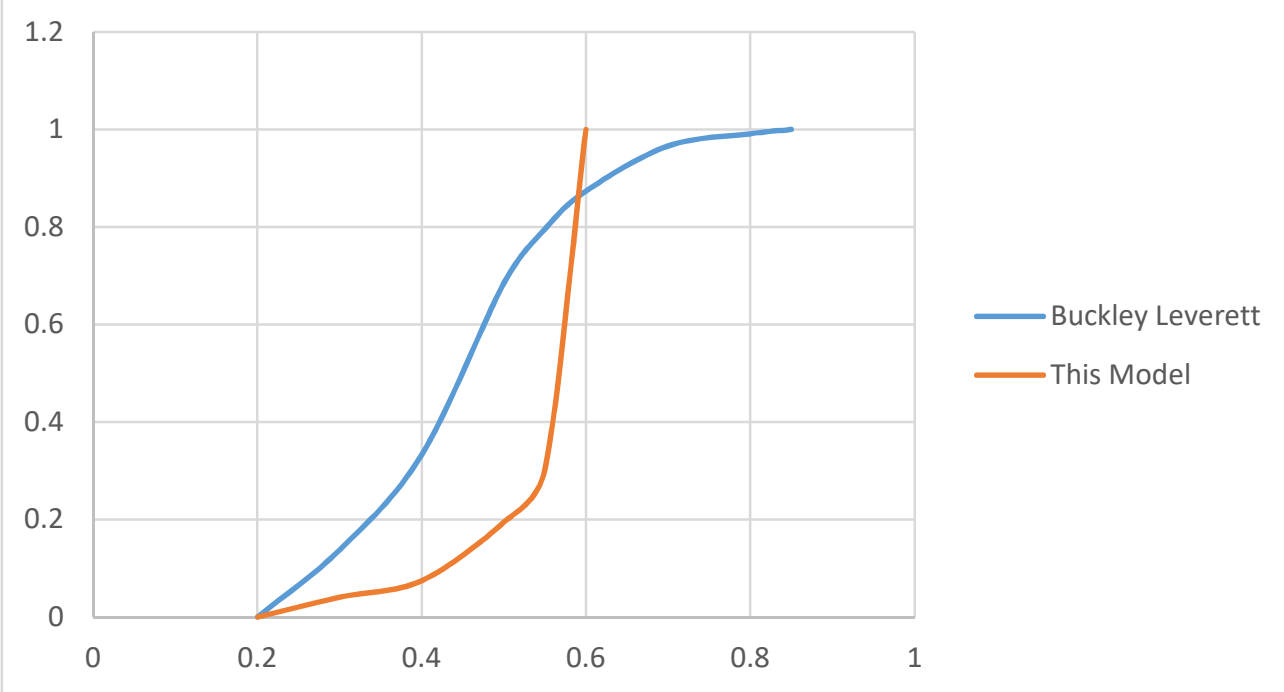

Fig. 2.0: Fractional flow of this model against the Buckley Leverett Method 


\section{NOMENCLATURE}

\begin{tabular}{|c|l|}
\hline SYMBOLS & DESCRIPTION \\
\hline$A$ & Effective pattern area, acres \\
\hline$f_{o}$ & Fraction of total flowing stream composed of oil \\
\hline$f_{w}$ & Fraction of total flowing stream composed of water \\
\hline $\mathrm{g}$ & Acceleration due to gravity, ft/sq sec \\
\hline$i_{w}$ & Fraction of total flowing stream composed of oil \\
\hline $\mathrm{k}$ & Formation absolute permeability \\
\hline$k_{o}$ & Effective permeability to oil, $\mathrm{md}$ \\
\hline$k_{w}$ & Effective permeability to water, $\mathrm{md}$ \\
\hline$k_{r o}$ & Relative permeability to oil, fraction \\
\hline$k_{r w}$ & Relative permeability to water, fraction \\
\hline$p_{c}$ & Capillary pressure $=\mathrm{P}_{0}-\mathrm{P}_{\mathrm{w}}=$ pressure in oil phase minus pressure in water phase \\
\hline $\mathrm{q}$ & Flow rate or production rate, B/D \\
\hline$\alpha_{d}$ & Angle of formation dip, degrees \\
\hline$\mu_{o}$ & Oil viscosity, $\mathrm{cP}$ \\
\hline$\mu_{w}$ & Water viscosity, $\mathrm{cp}$ \\
\hline$\rho_{0}$ & Oil density, gm/cc \\
\hline$\rho_{w}$ & Water density, gm/cc \\
\hline$\Delta \rho$ & Density difference, $\mathrm{w}$ \\
\hline$\Phi$ & ater density minus oil density, gm/cc \\
\hline
\end{tabular}

\section{CONCLUSION}

As evident from the result, the new fractional flow equation (equation 19.0) is proposed for a sandstone reservoir. This equation should be used in place of the usual fractional flow equation for non-Darcy flow. It is recommended that this work can be extended to other reservoir lithologies such as limestone or dolomite.

\section{REFERENCES}

1. Buckley, S. E. and Leverett, M. C.: "Mechanism of Fluid Displacements in Sands," Trans., AIME (1942).

2. Craig, F. F. : The Reservoir Engineering Aspects of Water flooding, SPE Monograph Series (1971). 